\title{
Adaptive mutations in bacteria: high rate and small effects
}

1. Lília Perfeito1,

2. Lisete Fernandes 1,2 ,

3. Catarina Mota1,

4. Isabel Gordo1,

\pm Author Affiliations

1. ${ }^{1}$ Instituto Gulbenkian de Ciência, Rua da Quinta Grande, number 6, 2780-156 Oeiras, Portugal.

2. ${ }^{2}$ Escola Superior de Tecnologia da Saúde de Lisboa, Lisboa, Portugal.

1. $\biguplus^{*}$ To whom correspondence should be addressed. E-mail: igordo@igc.gulbenkian.pt

- Abstract

Evolution by natural selection is driven by the continuous generation of adaptive mutations. We measured the genomic mutation rate that generates beneficial mutations and their effects on fitness in Escherichia coli under conditions in which the effect of competition between lineages carrying different beneficial mutations is minimized. We found a rate on the order of $10^{-5}$ per genome per generation, which is 1000 times as high as previous estimates, and a mean selective advantage of $1 \%$. Such a high rate of adaptive evolution has implications for the evolution of antibiotic resistance and pathogenicity.

- Received for publication 8 March 2007.

- Accepted for publication 2 July 2007.

Read the Full Text

\section{THIS ARTICLE HAS BEEN CITED BY OTHER ARTICLES:}

- Genomic mutation rates that neutralize adaptive evolution and natural selection $J R$ Soc Interface 29 May 2013: 20130329.
- Abstract
- Full Text
- Full Text (PDF)

- An ABC Method for Estimating the Rate and Distribution of Effects of Beneficial Mutations Genome Biol Evol 2 May 2013: 794-806.
- Abstract
- $\underline{\text { Full Text }}$ 
- Full Text (PDF)

- Long-term culture at elevated atmospheric $\mathrm{CO} 2$ fails to evoke specific adaptation in seven freshwater phytoplankton species Proc R Soc B 8 January 2013: 20122598.

- Abstract

- Full Text

- Full Text (PDF)

- Dynamic Population Changes in Mycobacterium tuberculosis During Acquisition and Fixation of Drug Resistance in Patients The Journal of Infectious Disease 1 December 2012: 1724-1733.

- $\underline{\text { Abstract }}$

- Full Text

- Full Text (PDF)

- Clonal Interference in the Evolution of Influenza Genetics 1 October 2012: 671-682.

- Abstract

- Full Text

- Full Text (PDF)

- Real time forecasting of near-future evolution $J$ R Soc Interface 7 September 2012: 2268-2278.

- Abstract

- $\underline{\text { Full Text }}$

- Full Text (PDF)

- Culture History and Population Heterogeneity as Determinants of Bacterial Adaptation: the Adaptomics of a Single Environmental Transition Microbiol. Mol. Biol. Rev. 1 September 2012: 597-625.

- Abstract

- Full Text

- Full Text (PDF)

- Cost of Antibiotic Resistance and the Geometry of Adaptation Mol Biol Evol 1 May 2012: 1417-1428.

- Abstract

- Full Text

- Full Text (PDF)

- Quantifying Selection Acting on a Complex Trait Using Allele Frequency Time Series Data Mol Biol Evol 1 April 2012: 1187-1197.

- Abstract

- Full Text

- Full Text (PDF)

- Distribution of fixed beneficial mutations and the rate of adaptation in asexual populations Proc. Natl. Acad. Sci. USA 27 March 2012: 4950-4955.

- Abstract

- Full Text

- Full Text (PDF)

- A method to infer positive selection from marker dynamics in an asexual population Bioinformatics 15 March 2012: 831-837.

- Abstract

- Full Text

- Full Text (PDF)

- Emergent Neutrality in Adaptive Asexual Evolution Genetics 1 December 2011: 13611375.

- Abstract

- Full Text

$\circ \quad$ Full Text (PDF) 
- Distinguishing Driver and Passenger Mutations in an Evolutionary History Categorized by Interference Genetics 1 November 2011: 989-1000.
- Abstract
- Full Text
- Full Text (PDF)

- Interfering Waves of Adaptation Promote Spatial Mixing Genetics 1 November 2011: 1045-1060.
$\circ$ Abstract
- Full Text
- Full Text (PDF)

- On measuring selection in experimental evolution Biol Lett 23 April 2011: 210-213.
- Abstract
- Full Text
- Full Text (PDF)

- Mutational Effects and Population Dynamics During Viral Adaptation Challenge Current Models Genetics 1 January 2011: 185-202.
- Abstract
- Full Text
- Full Text (PDF)

- Diminishing Returns From Beneficial Mutations and Pervasive Epistasis Shape the Fitness Landscape for Rifampicin Resistance in Pseudomonas aeruginosa Genetics 1 December 2010: 1345-1354.
- Abstract
- Full Text
- Full Text (PDF)

- Selective sweeps and parallel mutation in the adaptive recovery from deleterious mutation in Caenorhabditis elegans Genome Res 1 December 2010: 1663-1671.
- Abstract
- Full Text
- Full Text (PDF)

- Experimental Validation of the Predicted Binding Site of Escherichia coli K1 Outer Membrane Protein A to Human Brain Microvascular Endothelial Cells: IDENTIFICATION OF CRITICAL MUTATIONS THAT PREVENT E. COLI MENINGITIS J Biol Chem 26 November 2010: 37753-37761.
○ Abstract
- Full Text
- Full Text (PDF)

- Mutational Robustness of Ribosomal Protein Genes Science 5 November 2010: 825827.
- Abstract
- Full Text
- Full Text (PDF)

- Isolation of atypical enteropathogenic Escherichia coli from children with and without diarrhoea in Delhi and the National Capital Region, India J Med Microbiol 1 October 2010: 1156-1162.
- Abstract
- Full Text
- Full Text (PDF)

- A High Frequency of Beneficial Mutations Across Multiple Fitness Components in Saccharomyces cerevisiae Genetics 1 August 2010: 1397-1409.
- Abstract
- Full Text 
- Full Text (PDF)

- Escherichia coli rpoB Mutants Have Increased Evolvability in Proportion to Their Fitness Defects Mol Biol Evol 1 June 2010: 1338-1347.

- Abstract

- Full Text

- Full Text (PDF)

- The population genetics of beneficial mutations Phil Trans R Soc B 27 April 2010: 11951201.

- Abstract

- Full Text

- Full Text (PDF)

- Rate and effects of spontaneous mutations that affect fitness in mutator Escherichia coli Phil Trans R Soc B 27 April 2010: 1177-1186.

- Abstract

- Full Text

- Full Text (PDF)

- Beneficial mutations and the dynamics of adaptation in asexual populations Phil Trans R Soc B 27 April 2010: 1255-1263.

- Abstract

- $\underline{\text { Full Text }}$

- Full Text (PDF)

- Finite Populations, Finite Resources, and the Evolutionary Maintenance of Genetic Recombination J Hered 1 March 2010: S135-S141.
- Abstract
- Full Text
- Full Text (PDF)

- Genetic Architecture and the Evolution of Sex J Hered 1 March 2010: S142-S157.

- Abstract

- Full Text

- Full Text (PDF)

- Comparison of the Complete Genome Sequences of Bifidobacterium animalis subsp. lactis DSM 10140 and BI-04 J. Bacteriol. 1 July 2009: 4144-4151.
- Abstract
- Full Text
- Full Text (PDF)

- Clonal Interference, Multiple Mutations and Adaptation in Large Asexual Populations Genetics 1 December 2008: 2163-2173.
- Abstract
- Full Text
- Full Text (PDF)

- Surviving the Bottleneck: Transmission Mutants and the Evolution of Microbial Populations Genetics 1 December 2008: 2193-2200.

- Abstract

- Full Text

- Full Text (PDF)

- Selective Sweep at a Quantitative Trait Locus in the Presence of Background Genetic Variation Genetics 1 November 2008: 1645-1660.

- Abstract

- Full Text

- Full Text (PDF)

- Hitchhiking Both Ways: Effect of Two Interfering Selective Sweeps on Linked Neutral Variation Genetics 1 September 2008: 301-316. 
- Abstract

- Full Text

- Full Text (PDF)

- How much of protein sequence space has been explored by life on Earth? J $R$ Soc Interface 6 August 2008: 953-956.
$\circ$ Abstract
- Full Text
- Full Text (PDF)

- Synergistic Fitness Interactions and a High Frequency of Beneficial Changes Among Mutations Accumulated Under Relaxed Selection in Saccharomyces cerevisiae Genetics 1 March 2008: 1571-1578.
- Abstract
- Full Text
- Full Text (PDF)

- The effect of spatial structure on adaptation in Escherichia coli Biol Lett 23 February 2008: 57-59.
- Abstract
- Full Text
- Full Text (PDF)

- Clonal interference in large populations Proc. Natl. Acad. Sci. USA 13 November 2007: 18135-18140.
- Abstract
- Full Text
- Full Text (PDF) 


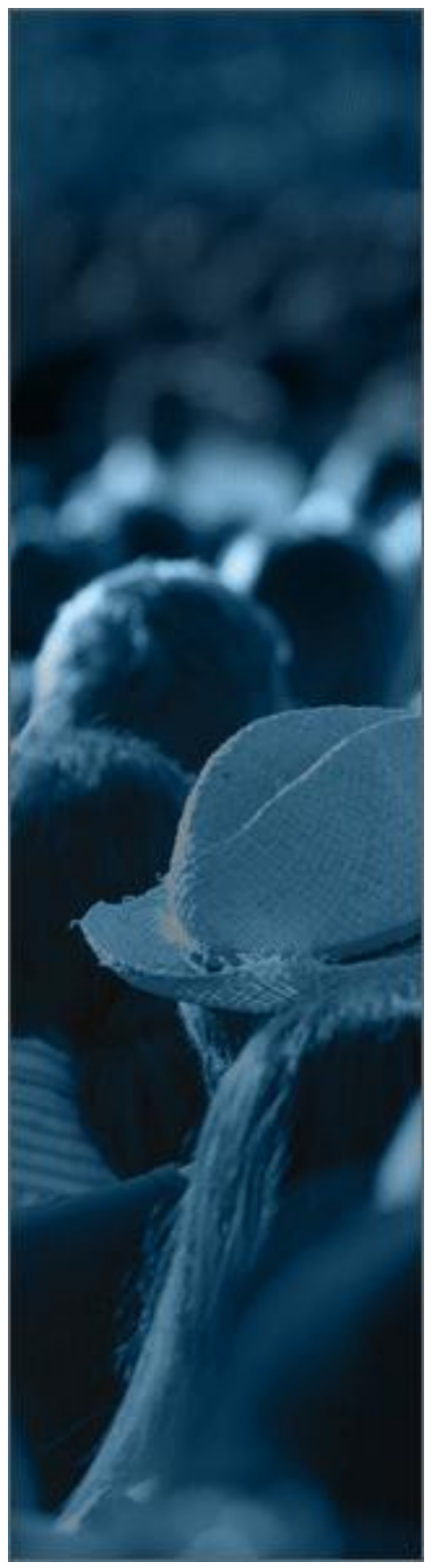

$\underline{\text { To Advertise Find Products }}$

Science. ISSN 0036-8075 (print), 1095-9203 (online) 